\title{
The making of a pest: Insights from the evolution of chemosensory receptor families in a pestiferous and invasive fly, Drosophila suzukii
}

\author{
Paul V. Hickner, Chissa L. Rivaldi, Cole M. Johnson, Madhura Siddappaji, Gregory J. Raster \\ and Zainulabeuddin Syed ${ }^{*}$ (i)
}

\begin{abstract}
Background: Drosophila suzukii differs from other melanogaster group members in their proclivity for laying eggs in fresh fruit rather than in fermenting fruits. Olfaction and gustation play a critical role during insect niche formation, and these senses are largely mediated by two important receptor families: olfactory and gustatory receptors (Ors and Grs). Earlier work from our laboratory has revealed how the olfactory landscape of D. suzukii is dominated by volatiles derived from its unique niche. Signaling and reception evolve in synchrony, since the interaction of ligands and receptors together mediate the chemosensory behavior. Here, we manually annotated the Ors and Grs in D. suzukii and two close relatives, D. biarmipes and D. takahashii, and compared these repertoires to those in other melanogaster group drosophilids to identify candidate chemoreceptors associated with D. suzukii's unusual niche utilization.
\end{abstract}

Results: Our comprehensive annotations of the chemosensory genomes in three species, and comparative analysis with other melanogaster group members provide insights into the evolution of chemosensation in the pestiferous D. suzukii. We annotated a total of 71 Or genes in D. suzukii, with nine of those being pseudogenes (12.7\%). Alternative splicing of two genes brings the total to 62 genes encoding 66 Ors. Duplications of Or23a and Or67a expanded D. suzukii's Or repertoire, while pseudogenization of Or74a, Or85a, and Or98b reduced the number of functional Ors to roughly the same as other annotated species in the melanogaster group. Seventy-one intact $\mathrm{Gr}$ genes and three pseudogenes were annotated in D. suzukii. Alternative splicing in three genes brings the total number of Grs to 81. We identified signatures of positive selection in two Ors and three Grs at nodes leading to D. suzukii, while three copies in the largest expanded Or lineage, Or67a, also showed signs of positive selection at the external nodes.

Conclusion: Our analysis of D. suzukii's chemoreceptor repertoires in the context of nine melanogaster group drosophilids, including two of its closest relatives (D. biarmipes and D. takahashii), revealed several candidate receptors associated with the adaptation of $D$. suzukii to its unique ecological niche.

Keywords: Olfaction, Gustation, Odorant receptors, Gustatory receptors, Niche utilization, Adaption, Molecular evolution, Episodic selection

\footnotetext{
* Correspondence: zainulabeuddin.syed.5@nd.edu

Department of Biological Sciences \& Eck Institute for Global Health,

University of Notre Dame, Notre Dame, IN 46556, USA
} 


\section{Background}

Chemoreception, broadly encompassing olfaction and gustation, is essential to a number of insect life history traits such as host detection and discrimination, mate location, and predator avoidance. Chemoreception in insects is largely mediated by two divergent protein families, olfactory receptors (Ors) and gustatory receptors (Grs). A third family described in 2009 by Benton et al. [1] as the ionotropic receptors (Irs) has been implicated in multiple sensory modalities, including chemosensation [2]. Insect chemoreceptors (Ors and Grs) are seven transmembrane proteins expressed on the surface of chemosensory neurons housed in hair-like structures called sensilla [3, 4]. The genome of Drosophila melanogaster contains 60 Ors encoding 62 proteins through alternative splicing $[4,5]$, and each $\mathrm{Or}$ is expressed in a specific sub-set of olfactory receptor neurons (ORNs), with very few exceptions. All ORNs expressing the same Or merge into a single glomerulus [6]. While the basic principles and mechanisms of olfaction remain conserved across phyla $[3,7,8]$, insect Ors have little homology to Caenorhabditis elegans or vertebrates, and the membrane topology is quite distinct [9]. Moreover, all canonical Ors are co-expressed with a single noncanonical olfactory receptor co-receptor (Orco), and together appear to define the response characteristic of an ORN [10]. The sense of taste in D. melanogaster is defined by 60 Grs encoding 68 proteins through alternative splicing [5]. In contrast to Ors, there is no clear evidence for a non-canonical co-receptor, and the membrane topology remains poorly defined [11].

The number of chemoreceptors often varies widely among insects, broadly reflecting their environment and function [3]. For example, the tsetse fly, Glossina morsitans, is estimated to have 40-46 Ors and 11-14 Grs $[12,13]$, while the red flour beetle, Tribolium castaneum, has 259 Ors and 220 Grs [14] (Tribolium Genome Sequencing Consortium 2008), and the honey bee, Apis mellifera, has 163 Ors and only 10 functional Grs [15]. The largest chemoreceptor repertoires (over 350 Ors) are reported in eusocial insects, such as ants [16]. In Drosophila, Or repertoires reflect the niche specialization patterns, such that a restricted spectrum of host/diet choice can be correlated with changes in chemoreceptor repertoire, such as specific losses and/or duplications in a set of receptors [17-21]. These changes can be further correlated with structural changes to the peripheral olfactory apparatus such as an altered number of specialized sensilla/ORNs [22-24]. Since, signaling and reception evolve in synchrony and in parsimony [25], an overall understanding of both these aspects will provide insights into the chemosensory basis of host utilization.

The recent (Drosophila 12 Genomes Consortium, Drosophila modENCODE Project) sequencing and subsequent annotation of multiple Drosophila spp. provides us with an excellent opportunity to connect the natural history of drosophilids [26] with the evolutionary history of chemosensation [27]. Recently, a member of the melanogaster group, Drosophila suzukii (Matsumura), has gained immense attention due to its invasion of the western hemisphere from its original endemic zone of South East Asia and emergence as a serious economic pest. A reduction in the yield of berry and soft fruit crops in newly invaded areas of North America and Europe are reported to reach as high as $80 \%$ in the absence of any management practices, although a current and comprehensive economic assessment is lacking [28, 29].

Among the Drosophilidae, comprising over 1,500 known species [30], D. suzukii is one of only a few Drosophila with a highly evolved serrated ovipositor [31] that enables gravid females to pierce the skin of fresh fruits and lay their eggs inside the flesh. Though D. suzukii has been recognized as a pest of cherries in Japan since 1931, they were found infesting strawberries and cranberries in California, USA in 2008 [29]. They have since been discovered in at least a dozen states in the USA, as well as areas of Canada, Mexico, Italy, Spain and France $[28,29,32]$. We recently conducted a comprehensive analysis of the suite of volatile organic chemicals (VOCs) that define the unique olfactory landscape of $D$. suzukii, and compared it with that of D. melanogaster [33]. We demonstrated that $D$. suzukii's unique attraction to fresh fruits may be associated with the distinctive volatile repertoire originating from the host fruit-fly associated yeast complex. Recent studies are providing exciting insights into the complex interactions of D. Suzukii with yeast and fruits [34].

Here, we explored the role of olfaction and gustation in D. suzukii's unique ecological niche. We first manually annotated the Ors and Grs in the recently sequenced $D$. suzukii genomes [35, 36], and two closely related species, D. biarmipes and D. takahashii (Drosophila modENCODE Project), herein collectively referred to as the suzukii-takahashii clade. The latter two species occur in geographically overlapping regions with D. suzukii [30] but are mostly saprophytic and do not have the pointed ovipositor that enables them to lay eggs in fresh fruits [31]. We then compared these repertoires to those in six other previously annotated melanogaster group Drosophila [5, 19, 37]. Following our earlier comprehensive analysis of ligand repertoires for D. suzukii [33], we present the associated chemoreceptor repertoire that together defines D. suzukii's unique ability to exploit diverse niches, and in turn pose a serious threat to fruit crops. This study further adds to ongoing efforts in understanding the chemosensory basis of host and mate finding in D. suzukii [33, 34, 38-40]. 


\section{Methods}

\section{Manual curation of Or and Gr repertoires}

D. suzukii gene models were manually curated based on the D. melanogaster $\mathrm{Or}$ and $\mathrm{Gr}$ annotations in FlyBase version FB2015_03 [41]. In short, D. melanogaster peptide sequences were used to screen the D. suzukii genome scaffolds using tBLASTn analysis in SpottedWingFlyBase v1.0 (last accessed on 4 September, 2015) [35]. To help predict start and stop codons, and exonintron boundaries, scaffold regions containing putative chemoreceptors were aligned with their homologous $D$. melanogaster coding sequences (CDS) in MultAlin [42]. Where exon-intron boundaries were ambiguous, intron donor and accepter sites were evaluated using the splice site prediction tool [43] on the Berkeley Drosophila Genome Project web site (http://www.fruitfly.org/seq tools/splice.html). Complementary strands were generated using the Reverse Compliment tool in the Sequence Manipulation Suite [44] (http://www.bioinformatics.org/ sms/rev_comp.html), and coding sequences were translated using the ExPASy translate tool [45]. The D. suzukii Or and $\mathrm{Gr}$ annotations were then used to screen the D. biarmipes (Dbia_2.0, GCA_000233415.2) and D. takahashii (Dtak_2.0, GCA_000224235.2) genome assemblies with the methods described for $D$. suzukii using the BLAST tools on the National Center for Biotechnology Information (NCBI) web server. The D. biarmipes and D. takahashii genome assemblies were generated and made publicly available by the Drosophila ModENCODE project and the Baylor College of Medicine-Human Genome Sequencing Center (BCM-HGSC).

\section{Gap filling and sequence validation Gap filling}

We filled gaps in the genome scaffolds that prevented the building of complete gene models using the sequence read archive (SRA) databases in NCBI. In those gene models where this method failed, PCR and capillary sequencing were used to fill the gaps.

\section{Validation of duplications}

Two approaches were used to evaluate duplications. When possible, tandem repeats were confirmed by amplifying and sequencing a region spanning the proximal ends of the duplicates. However, when the copies were greater than $\sim 4,000$ nucleotides apart or on a different scaffold we sequenced the individual genes.

\section{Validation of pseudogenes}

Predicted pseudogenes were resequenced to confirm the predictions from the initial tBLASTn analysis for D. suzukii, D. takahashii and D. biarmipes.
Genomic DNA (gDNA) for resequencing was extracted from the strains used for genome sequencing that are presently available at the UC San Diego Drosophila Stock Center: D. suzukii (stock \# 14023-0311.03), D. biarmipes (stock \# 14023-0361.10) and D. takahashii (stock \# 14022-0311.13). A cetyltrimethylammonium bromide (CTAB) protocol [46] was modified for the extraction of genomic DNA from insects. Ten adult flies (5 males and 5 females) were ground with a pestle in $1.5 \mathrm{ml}$ microcentrifuge tubes containing $200 \mu \mathrm{l} 2 \% \mathrm{CTAB}$ solution $(100 \mathrm{mM}$ Tris $\mathrm{HCl} \mathrm{pH} \mathrm{8.0,} 10 \mathrm{mM}$ EDTA, $1.4 \mathrm{M} \mathrm{NaCl}$, and $2 \%$ CTAB). Samples were incubated for $5 \mathrm{~min}$ at $65{ }^{\circ} \mathrm{C}$, followed by the addition of $200 \mu \mathrm{l}$ chloroform and mixing by inverting 10 times. Samples were then centrifuged for $5 \mathrm{~min}$ at 13,000 $\mathrm{x}$ g. The aqueous phase was removed and placed in a new tube containing $200 \mu \mathrm{l}$ isopropanol, mixed by inverting 10 times, and centrifuged for $5 \mathrm{~min}$ at 13,000 $\mathrm{x}$ g. The supernatant was poured off, $500 \mu \mathrm{l}$ of $70 \%$ ethanol was added, and the sample was centrifuged for $5 \mathrm{~min}$ at $13,000 \times \mathrm{g}$. The supernatant was removed and the pellet was allowed to dry at room temperature for $15 \mathrm{~min}$. DNA was resuspended in $50 \mu \mathrm{l}$ deionized water, and all samples were normalized to $50 \mathrm{ng} / \mu \mathrm{l}$ using a Nanodrop ND-2000 (ThermoScientific, USA).

Primers flanking the gaps were designed using the Primer3plus program [47]. PCR was carried out in $50 \mu \mathrm{l}$ reaction volumes using $\mathrm{GoTaq}^{\odot}$ reagents (Promega). Each reaction contained a final concentration of $0.2 \mu \mathrm{M}$ of each primer, 1.0 units of Taq polymerase and $2 \mathrm{ng} / \mu \mathrm{l}$ of genomic DNA. The thermal cycle included an initial denaturation of $94{ }^{\circ} \mathrm{C}$ for $2 \mathrm{~min}, 35$ cycles of $94{ }^{\circ} \mathrm{C}$ for $1 \mathrm{~min}, 58^{\circ}$ $\mathrm{C}$ for $1 \mathrm{~min}$, and $72{ }^{\circ} \mathrm{C}$ for $2 \mathrm{~min}$, with a final extension time of $5 \mathrm{~min}$ at $72{ }^{\circ} \mathrm{C}$. PCR products were visualized with agarose gel electrophoresis using $\mathrm{SYBR}^{\bullet}$ Safe gel stain (ThermoScientific). PCR products were cleanedup using the Wizard ${ }^{\circledR}$ SV Gel and PCR Clean-Up System (Promega). Sequencing was performed using the ABI 3730xl (Life Technologies) and BigDye ${ }^{\varpi}$ chemistry (Life Technologies) at the University of Notre Dame Genomics Core Facility. Genes with sequence gaps filled using the SRA databases or by PCR and sequencing were suffixed with "fixSRA" or "fixPCR", respectively. Nucleotides that were fixed based on SRA or PCR are in bold or underlined, respectively (Additional file 1: Table S1-S6).

\section{Gene nomenclature}

Ors and Grs were named based on homology to D. melanogaster using standard Drosophila community gene nomenclature [48]. Each gene was prefixed with 'D' and the first three letters of the specific epithet (Dsuz, Dbia or Dtak), and named based on a combination of phylogenetic and reciprocal BLASTp analyses with the $D$. melanogaster annotated protein database in FlyBase 
version FB2015_03 [41]. Duplications were suffixed with a unique numeral (e.g. DsuzOr23a-1 and DsuzOr23a-2). Splice variants were predicted solely on genomic sequence (no transcript evidence) and suffixed using the capital letter designation in accordance with the homologous splice variant in FlyBase for $D$. melanogaster (e.g. DmelOr69aA and DsuzOr69aA). However, where novel splice variants were predicted, splice variants were designated based on their order on the scaffold rather than homology to $D$. melanogaster.

Pseudogenes were suffixed with 'P', and are defined here as genes with a mutated start codon, premature stop codon, or frameshift mutation leading to loss of $\geq 20 \%$ of the original protein and $\geq 1$ transmembrane domain [19] compared to the $D$. melanogaster homolog. The number of transmembrane domains was predicted using the topology prediction program, OCTOPUS [49]. Pseudogenes that were not excessively degraded were reconstructed for phylogenetic analysis by repairing mutated start codons, exon-intron boundaries or frameshift mutations to a functional state based on an intact homolog in the suzukii or takahashii subgroup. Repaired nucleotides are in lowercase in Table S1. All genes other than pseudogenes and partial gene models are assumed to be functional and are referred to here as intact. We refer to a lineage as lost when pseudogenizations or deletions (no apparent vestiges) resulted in the absence of at least one intact gene in one of $47 \mathrm{Gr}$ or $54 \mathrm{Or}$ orthologous groups (OGs) present in the melanogaster group as defined by Almeida et al. 2014 [50] (see Additional file 2: Table S3 and S4).

Comparisons were made to the previously annotated chemoreceptor repertoires of $D$. melanogaster [5], D. ananassae, D. erecta, D. sechellia, D. simulans, and D. yakuba $[19,37]$. To better characterize lineages that were lost in the suzukii-takahashii clade, we screened the genomes of six additional Drosophila genome assemblies (D. bipectinata, D. elegans, D. eugracilis, D. ficusphila, D. kikkawai, and $D$. rhopaloa), generated and made publicly available by the BCM-HGSC, using the methods described above. Evolutionary inferences were based on phylogeny reconstruction by Chiu et al. [35], while divergence times were based on earlier estimates [36]. Reconciliation of gene trees with the species tree for the expanded lineages was performed using the parsimony-based method in NOTUNG v2.8.1.6 [51]. Gene trees were estimated using Mega version 6 [52] where the maximum likelihood approach with the Jones, Taylor, Thornton (JTT) substitution model [53], a Gamma distribution $(+G)$ with five discrete categories, and complete deletion of gaps was implemented. The edge weight thresholds were 0.9 and based on bootstrap support following 500 iterations, while the loss and duplication costs were 1.0 and 1.5 , respectively. No branches were collapsed for NOTUNG analysis.

\section{Measures of divergence}

Two proxies were used to describe divergence, the percent of identical amino acids in a peptide sequence alignment to $D$. melanogaster (\%ID) and the ratio of nonsynonymous $(d N)$ to synonymous $(d S)$ substitution rates $(d N / d S)$. \%ID was calculated using Clustal Omega [54] on the European Bioinformatics Institute (EMBLEBI) web server [55]. Nonsynonymous and synonymous substitution rates were calculated using the Nei and Gojobori method [56] implemented in SNAP v2.1.1 [57] (http://www.hiv.lanl.gov/content/sequence/SNAP/

SNAP.html). D. melanogaster was used as an outgroup for $d N$ and $d S$ calculations for all three species (i.e. suzukii-melanogaster, biarmipes-melanogaster, and takahashii-melanogaster). Differences were determined using paired (between species) and unpaired (Ors vs Grs) Wilcoxon Signed-Rank tests with the MASS package [53] in the R statistical environment.

\section{Tests for positive selection}

Positive selection acting on a small proportion of sites is often hard to detect using the ratio of nonsynonymous to synonymous substitution rates across the entire length of a gene $(d N / d S)$. Therefore, we used the adaptive branch-site random effects likelihood (aBSREL) approach [52] to identify signatures of diversifying selection at the codon level within a phylogenetic framework comprised of 9 species in the melanogaster group: $D$. ananassae, $D$. biarmipes, $D$. erecta, $D$. melanogaster, D. sechellia, D. simulans, D. suzukii, D. takahashii and D. yakuba. Or sequences for $D$. ananassae, D. erecta, D. sechellia, D. simulans and D. yakuba were from Guo and Kim [37] while Gr sequences were kindly provided by Michael Ritchie (University of St Andrews, UK). Only functional genes were used in the positive selection analysis.

Peptide sequences of homologous chemoreceptors (gene sets) were aligned in MAFFT v7 using the Blosum62 scoring matrix, a gap penalty of 1.53 , and the G-INS-1 refinement method [58]. Each alignment was visually inspected and manually edited, when necessary, and used to estimate a phylogeny for each homologous gene set. The maximum likelihood approach with the Jones, Taylor, Thornton (JTT) substitution model [59] and a Gamma distribution $(+\mathrm{G})$ with five discrete categories, and complete deletion of gaps was implemented in Mega version 6 [60]. Codon alignments were generated using PAL2NAL [61]. The aBSREL method [52] was implemented in HyPhy [62], where all internal and external nodes were tested for signatures of diversifying selection using likelihood ratio tests (LRTs). The HolmBonferroni method was used to control the familywise error rate for multiple tests within a gene set [63], whereas the Benjamini-Hochberg false discovery rate 
method was used for corrections across all gene sets [64]. Chemoreceptors showing positive selection based on the aBSREL method were further tested by using the stringent M1-M2 models of the codeml program in PAML [65]. Values >0.95 from Bayes empirical Bayes (BEB) method were considered sites under diversifying selection [66].

\section{Phylogenetic analysis}

Phylogenies were estimated for Ors and Grs to help reconstruct evolutionary events and to assist in the naming of the genes. Peptide sequences of $D$. suzukii, D. biarmipes, D. takahashii and D. melanogaster $\geq 360$ aa (Ors) or $\geq 340$ (Grs) in length were multiply aligned using MUSCLE v3.8.31 [67]. Maximum likelihood trees were inferred using the PROTGAMMA model of protein substitution, JTT matrix, and 500 bootstrap replications in RAxML v.8 [68]. RAxML analysis was conducted on the CIPRES Science Gateway and XSEDE [69]. Figures were prepared using the FigTree program for visualization and annotation of phylogenetic trees [70]. The $\mathrm{Or}$ and $\mathrm{Gr}$ trees were rooted with Orco and Gr21a, respectively. The aligned peptide sequences files (Phylip) and phylogenetic tree files (Nexus) for both the OR and $\mathrm{Gr}$ families are in the additional files (Additional files 3, 4, 5 and 6)

\section{Scanning electron microscopy (SEM)}

Freshly emerged D. suzukii were placed in acetone for at least $24 \mathrm{~h}$ until they could be processed by scanning electron microscopy (SEM). After undergoing critical point drying, flies were mounted both dorsally and ventrally on carbon tape attached to an aluminum stub mount, and coated with $4 \mu \mathrm{M}$ of iridium using a Cressington 208 HR sputter coater (Cressington Scientific Instruments, Watford, UK) in conjunction with the Cressington MTM 20 thickness monitor. Images were taken with a FEI-Magellan 400 FESEM (FEI, Hillsboro, OR, USA).

\section{Results}

\section{Chemosensory organs and receptor repertoires}

Scanning Electron Microscopy (SEM) of the olfactory organs in D. suzukii revealed striking morphological similarity to the well-defined D. melanogaster structures (Fig. 1) [71, 72]. Maxillary palps were adorned with a single class of olfactory sensilla, basiconic (Fig. 1c, d), whereas an additional two types, trichodea and coeloconic, are seen on the antenna (Fig. 1e). One unusual feature we noted in the large basiconic class was the presence of two distinct pore patterns. The single pattern reported earlier in D. melanogaster (Fig. 1f; circle) $[71,72]$ was observed in D. suzukii, but we also noted an additional unique pore pattern (Fig. 1f; circle).

Next, we annotated the Ors and Grs from the genome assemblies of $D$. suzukii and two closely related members, D. biarmipes and D. takahashii. A summary of the Or and $\mathrm{Gr}$ repertoires, along with those previously annotated in D. melanogaster, are reported in Table 1. Phylogenetic relationships among the Ors in these four species are represented in Fig. 2, illustrating several clade specific and species specific expansions. The total number of Or loci ranged from 64 in D. biarmipes to 71 in D. suzukii and D. takahashii. However, pseudogenizations reduced the
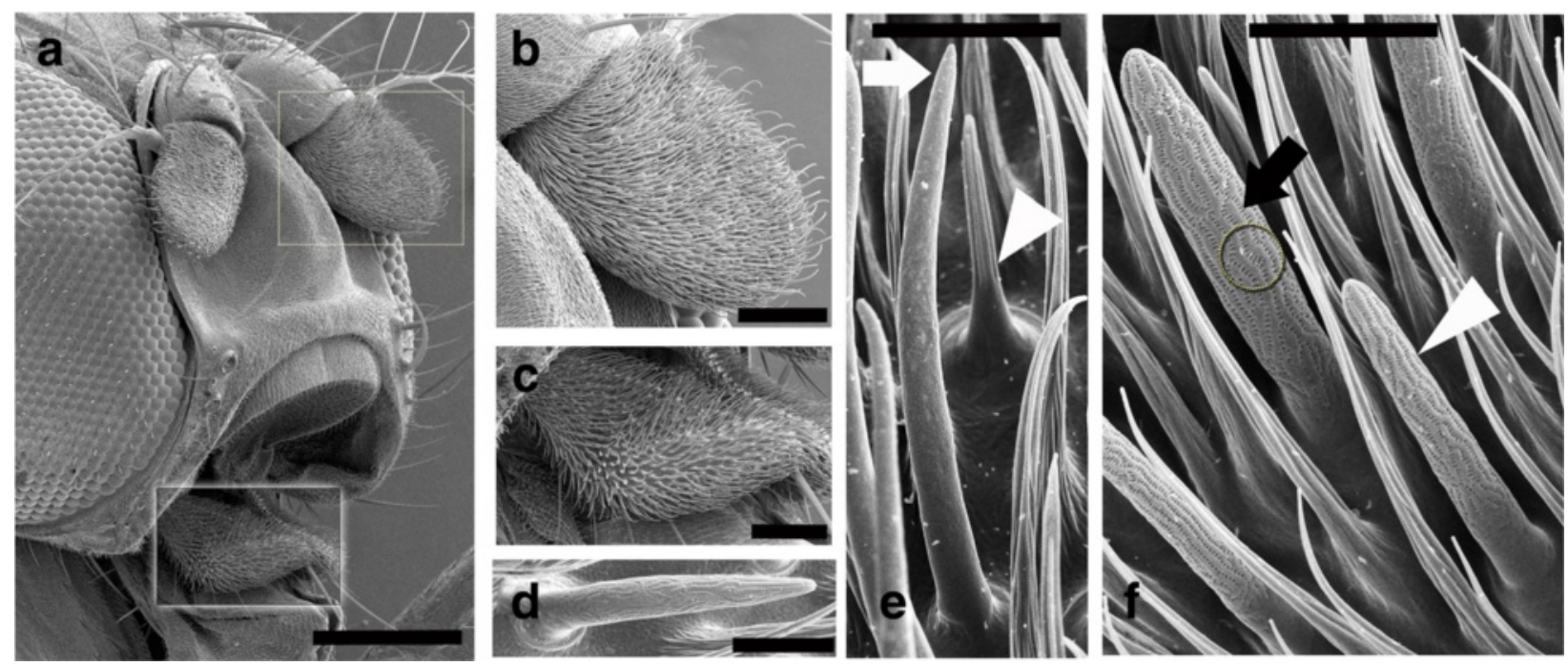

Fig. 1 Olfactory structures in D. suzukii viewed under SEM. An adult head with antenna and maxillary palp, highlighted in the insets. a Magnified antenna (b) and a maxillary palp (c). Palps have only one type of multiporous basiconic sensillum type (d). Antennal surface shows: long pointed trichoid sensillum (white arrow) and distinct coeloconic (arrow head) (e); multiporous basiconic sensilla (black arrow points to the large basiconic and short basiconic are indicated by white arrow head) (f). Scale bar is $200 \mu \mathrm{M}$ in (a), $50 \mu \mathrm{M}$ in (b) and (c); $5 \mu \mathrm{M}$ for (d), (e) and (f) 
Table 1 Summary of the chemoreceptor repertoires in D. suzukii (Dsuz), D. biarmipes (Dbia), and D. takahashii (Dtak), along with those previously annotated in D. melanogaster (Dmel)

\begin{tabular}{|c|c|c|c|c|c|c|c|c|}
\hline & \multicolumn{4}{|l|}{ ORs } & \multicolumn{4}{|l|}{ GRs } \\
\hline & Dsuz & Dbia & Dtak & Dmel $^{*}$ & Dsuz & Dbia & Dtak & Dmel* $^{*}$ \\
\hline$\overline{\text { Loci }}$ & 71 & 64 & 71 & 62 & 74 & 74 & 88 & 62 \\
\hline Functional genes & 62 & 60 & 70 & 60 & 71 & 74 & 82 & 60 \\
\hline Pseudogenes & 9 & 4 & 1 & 2 & 3 & 0 & 6 & 2 \\
\hline Genes w/splice variants & 2 & 2 & 2 & 2 & 3 & 3 & 3 & 3 \\
\hline Splice variants & 6 & 7 & 9 & 4 & 13 & 12 & 12 & 11 \\
\hline Total functional proteins & 66 & 65 & 77 & 62 & 81 & 83 & 91 & 68 \\
\hline
\end{tabular}

Total functional proteins include predicted splice variants

*Data from $[5,77,97-99]$

number of functional Or genes to 62, 60, and 70 in $D$. suzukii, $D$. biarmipes and $D$. takahashii, respectively. We predicted alternative splicing in two Or genes (Or46a and Or69a) in all three species, the same genes with splice variants in D. melanogaster [5]. Or46a encodes two splice variants that are moderately conserved, with percent identity of D. suzukii to D. melanogaster ranging from 80.7 to 83.4 \% (Additional file 1: Table S1). Conversely, Or69a is predicted to encode four to seven splice variants in the suzukii-takahashii clade, compared to only two isoforms in $D$. melanogaster. The number of functional genes in $D$. suzukii and D. biarmipes is roughly the same as D. melanogaster, whereas $D$. takahashii, with 70 genes, is more than the 66 predicted in $D$. ananassae, the largest Or repertoire among the melanogaster group Drosophila annotated prior to this study.

We predicted a total of $74 \mathrm{Gr}$ genes in D. suzukii, of which 71 are functional and three are pseudogenes, while 74 intact genes and no pseudogenes were predicted in $D$. biarmipes, and 88 genes were predicted in D. takahashii of which six are pseudogenes (Table 1). Phylogenetic relationships among the Grs in four species showed several unique expansions (Fig. 3). In D. suzukii, three genes encode 13 splice variants, bringing the total to 81 functional Grs (Table 1). D. suzukii's repertoire of Grs is nearly identical to D. biarmipes, which has 74 genes encoding $83 \mathrm{Grs}$. While these two $\mathrm{Gr}$ repertoires are larger than any other Drosophila annotated thus far, D. takahashii's repertoire is even larger with 82 intact genes encoding 91 Grs (Table 1).

The number of introns in Ors and Grs was consistent with those in D. melanogaster, with the exception of Gr85a. D. suzukii, D. biarmipes and D. takahashii each have two copies of Gr85a, and Gr85a-1 has one intron while Gr85a-2 has two introns. Furthermore, the peptide sequences are notably shorter (374-381 aa) than Gr85a in D. melanogaster (397 aa). The functional state of Or42a in both $D$. suzukii and D. biarmipes was initially unclear due to an unusually long first intron. Or42a resides on two different scaffolds in both species where it is fragmented in the $1^{\text {st }}$ intron. Attempts to amplify and sequence the gene region were unsuccessful. Or42a in D. takahashii has a large first intron (2511 nucleotides) compared to $D$. ananassae (66 nucleotides) and D. melanogaster (185 nucleotides), so next we examined Or42a in other melanogaster group genomes and found that the first intron is also large in D. kikkawai $(4,475)$, and on two different scaffolds in the D. eugracilis assembly. Consequently, failure to amplify the gene could have been due to the size of the amplicon. Screening of the SRA from transcriptome sequencing by Chiu et al. [35], however, shows that $\mathrm{Or} 42 a$ is being transcribed in $D$. suzukii; therefore, we considered $\operatorname{Or} 42 a$ intact in $D$. suzukii and D. biarmipes.

\section{Evolutionary events \\ Expansions and losses}

Gene tree reconciliation revealed complex birth-anddeath evolutionary patterns, wherein the suzukii and takahashii subfamilies (Fig. 4a and b; shaded box) underwent changes in copy numbers in a subset of Ors and Grs as they diverged from their common ancestor (CA1 in Fig. 4a). The later split of D. suzukii and D. biarmipes from CA2 was accompanied by similar changes. Three Or lineages, Or74a, Or85a and Or98b were lost in $D$. suzukii but were functional in $D$. biarmipes and D. takahashii, while Or33c was lost in D. biarmipes, and none were lost in D. takahashii (Fig. 4). Based on previous annotations, and the screening of five additional melanogaster group genomes, the loss of Or74a is unique to D. suzukii, while Or85a was lost independently in $D$. ananassae and D. suzukii.

The two largest expansions in the D. suzukii and $D$. takahashii Or lineages were Or23a and Or67a (Fig. 4b; Additional file 7: Figure S1). D. suzukii and D. takahashii have four and five copies of Or23a, respectively, while $D$. biarmipes has only one (Fig. 4b). Four intact and one Or67a pseudogene were found in D. suzukii, while four intact copies were found in D. biarmipes, and six copies plus a pseudogene were found in D. takahashii (Fig. 4b). The 


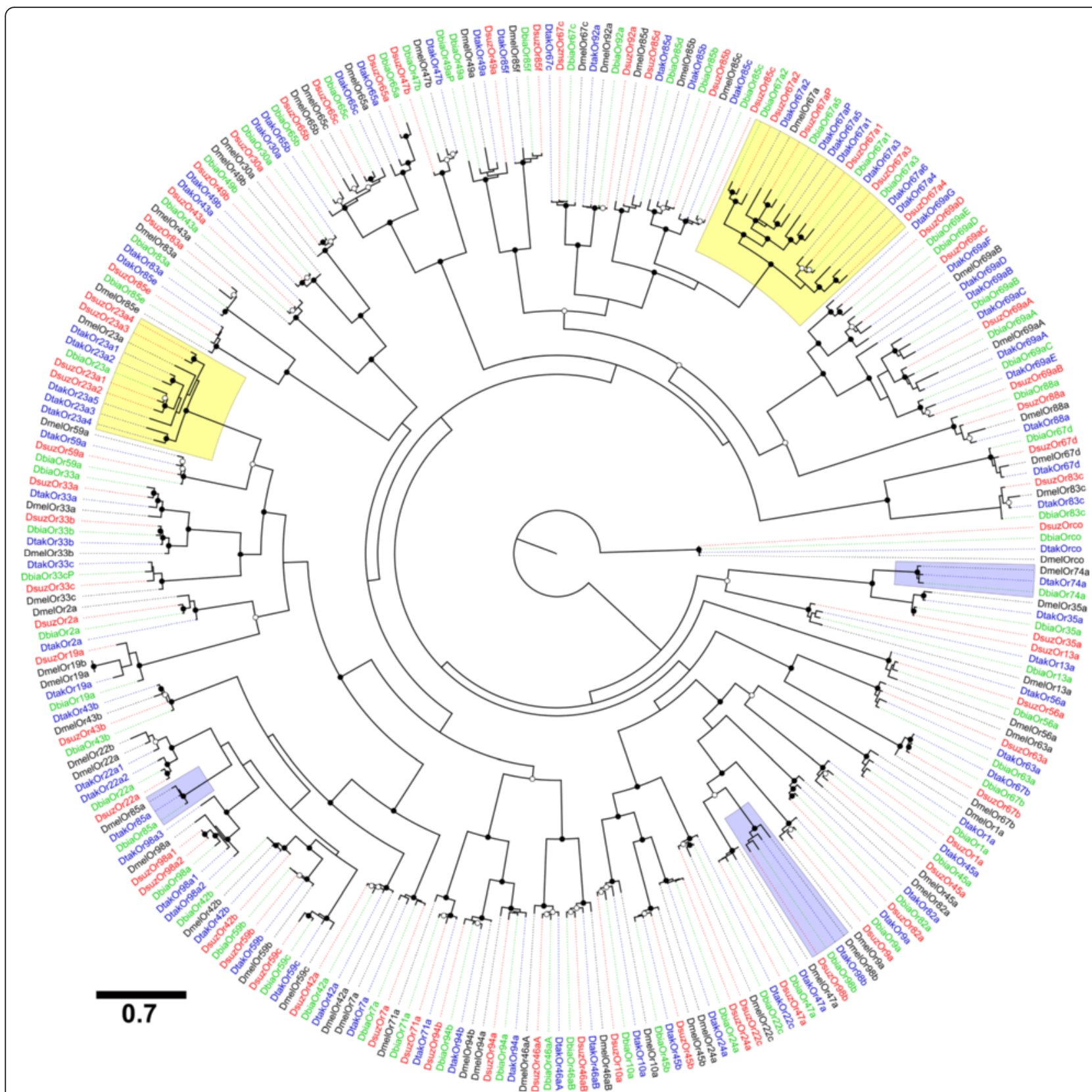

Fig. 2 Phylogenetic analysis of Ors in four Drosophila using a Maximum Likelihood method. The evolutionary history was inferred by using the Maximum Likelihood method based on the JTT matrix-based model [59]. The tree was constructed using RAxML under the JTT model of substitution with NNI topology search [68], based on an amino acid alignment by MUSCLE [67]. Branch support was estimated using 500 bootstrap replications. Expanded and lost lineages in D. suzukii are highlighted in yellow and blue, respectively

Gr lineages showed by far the largest expansions in the suzukii-takahashii clade compared to all of the annotated melanogaster group Drosophila. Four lineages were expanded in D. suzukii, two in D. biarmipes, and six in D. takahashii (Fig. 4a and b; Additional file 7: Figure S1). One lineage, Gr59cd, was expanded in all three members of the suzukii-takahashii clade, whereas Gr36a-c was uniquely expanded in the D. suzukii and D. biarmipes. The only other shared expansion was between
D. suzukii and D. takahashii for Gr59ab. The largest number of unique expansions in the suzukii-takahashii clade was in D. takahashii and includes Gr22a-f, Gr64a, Gr64f and $G r 98 b-d$. Interestingly, no $G r$ lineages were lost in any of the three species annotated in the present study.

Next, we used the parsimony-based gene tree reconciliation method in NOTUNG v2.8.1.6 [51] to analyze the two largest expanded lineages in both Ors and Grs. Among the Ors, Or23a duplicated 


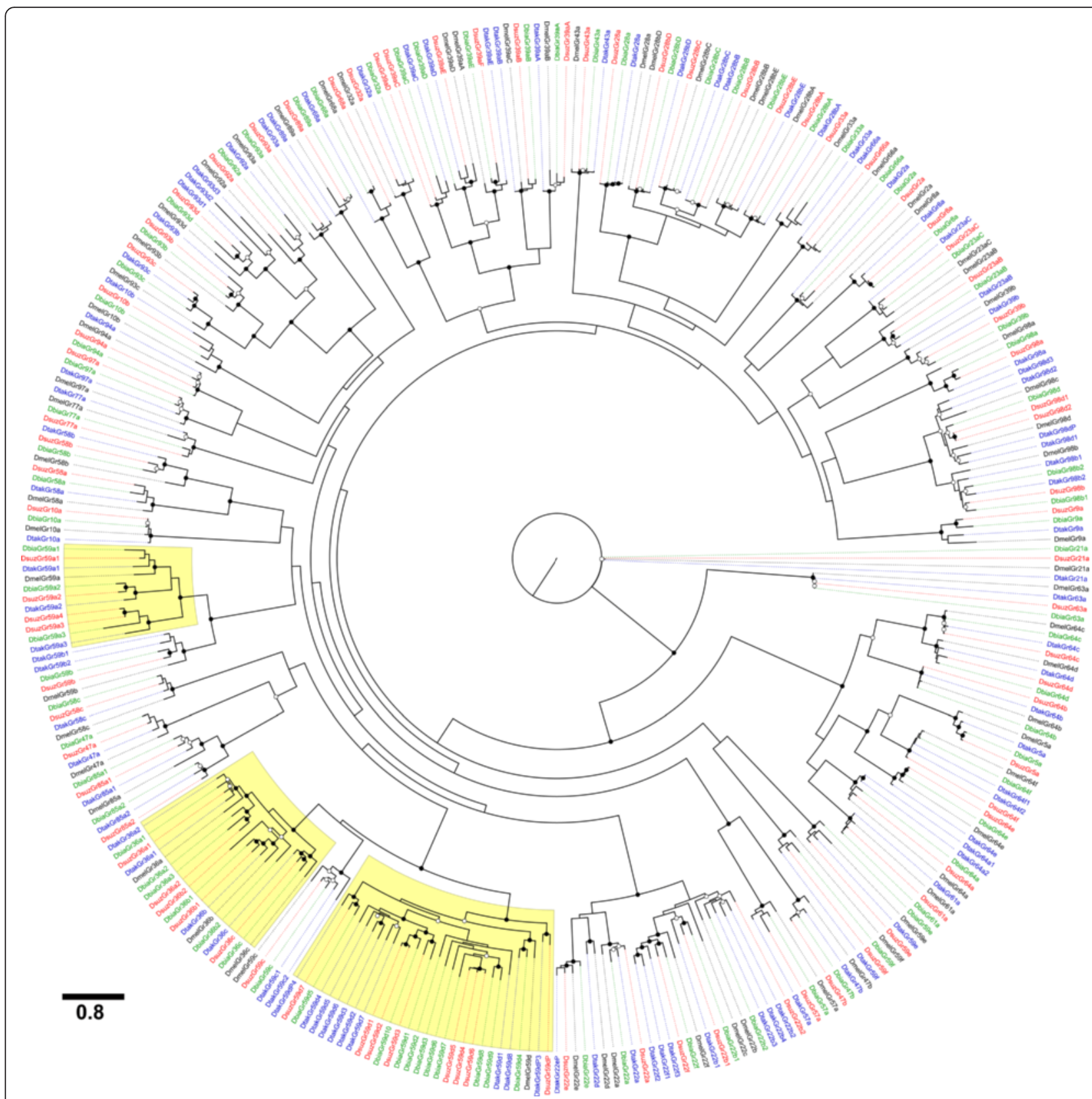

Fig. 3 Phylogenetic analysis of Grs in four Drosophila using a Maximum Likelihood method. The evolutionary history was inferred by using the Maximum Likelihood method based on the JTT matrix-based model [59]. The tree was constructed using RAxML under the JTT model of substitution with NNI topology search [68], based on an amino acid alignment by MUSCLE [67]. Branch support was estimated using 500 bootstrap replications. Expanded lost lineages in D. suzukii are highlighted in yellow

several times, and the common ancestor to the suzukii-takahashii clade probably had three copies, indicating that $D$. biarmipes lost two copies while $D$. suzukii and D. takahashii gained one and two copies, respectively (Additional file 7: Figure S1). The expansion of the Or67a lineage was already present prior to the suzukii-takahashii split, except for one later duplication in D. takahashii (Fig. S1). The two largest expanded $G r$ lineages were Gr59a and Gr59d in all three species. The Gr59a duplication pattern was comparable to Or67a, whereas Gr59d showed by far the most complex pattern of evolution resulting in 27 total copies in the three species (Fig. S1).

\section{Divergence}

Having annotated the genomes of three species that include the pest, $D$. suzukii, we estimated divergence and 


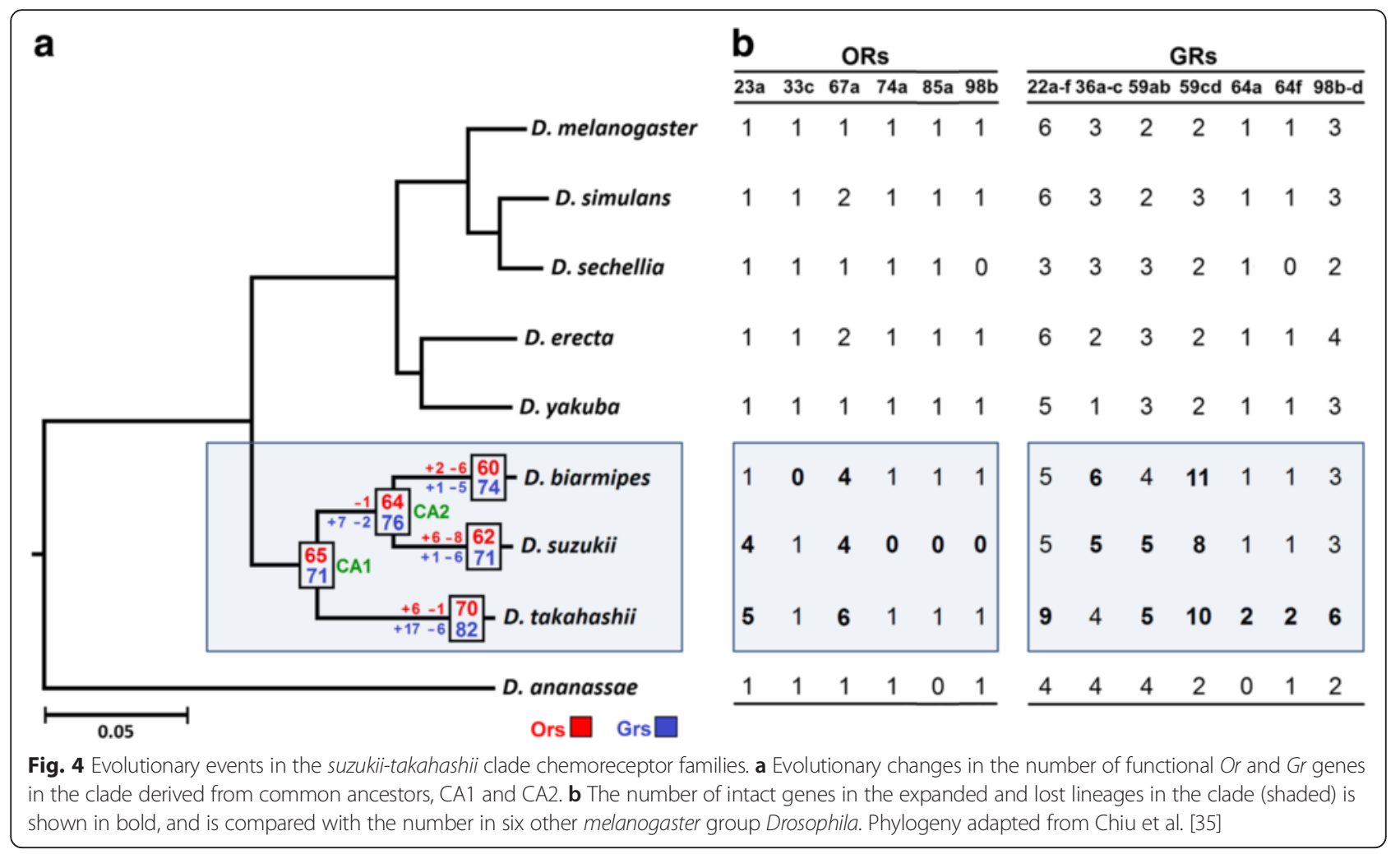

selection in the chemosensory receptor families using the percent of identical amino acids to homologous $D$. melanogaster peptide sequences (\%ID) and the ratio of nonsynonymous $(d N)$ to synonymous $(d S)$ substitution rates $(d N / d S)$ (Table 2). The $d N / d S$ ratios in Ors ranged from 0.0125 in DsuzOrco to 0.3670 in DbiaOr19a (mean of three species $=0.111$ ), while the \%IDs ranged from $44.72 \%$ in DbiaOr67a-2 to $98.77 \%$ in DsuzOrco $($ mean $=81.9 \%)$. The \%ID of Grs ranged from $33.06 \%$ in DbiaGr59a-3 to $99.55 \%$ in Gr21a (mean $=74.8 \%$ ). The $\mathrm{Gr}$ dN/dS ratios ranged from 0.002 in Gr21a to 0.370 in Gr10b (mean $=0.138$ ). These low $d N / d S$ values imply that both chemoreceptor families have evolved under strong purifying selection. These values are larger than the reported genome wide estimates of 0.095 for $\mathrm{X}$

Table 2 Substitution rate analysis of Ors and Grs

\begin{tabular}{|c|c|c|c|c|c|c|c|}
\hline \multirow[b]{2}{*}{ Genes } & \multirow[b]{2}{*}{ Parameter } & \multicolumn{3}{|c|}{ species } & \multicolumn{3}{|c|}{$p$-values } \\
\hline & & Dsuz & Dbia & Dtak & suz-bia & suz-tak & bia-tak \\
\hline \multirow[t]{3}{*}{ Ors } & $d N$ & 0.094 & 0.103 & 0.089 & 0.023 & 0.001 & $<0.001$ \\
\hline & $d S$ & 0.936 & 1.009 & 0.945 & 0.007 & 0.790 & 0.048 \\
\hline & $d N / d S$ & 0.102 & 0.104 & 0.093 & 0.679 & 0.006 & 0.009 \\
\hline \multirow[t]{3}{*}{ Grs } & $d N$ & 0.146 & 0.152 & 0.141 & 0.000 & 0.004 & $<0.001$ \\
\hline & $d S$ & 1.036 & 1.039 & 1.018 & 0.373 & 0.489 & 0.707 \\
\hline & $d N / d S$ & 0.141 & 0.142 & 0.131 & 0.003 & 0.008 & 0.014 \\
\hline
\end{tabular}

Differences in mean nonsynonymous $(d N)$ and synonymous $(d S)$ substitution rates, and $d N / d S$ are indicated by asterisks chromosome genes and 0.090 for autosomal genes [35]. Differences in the means between species, based on paired Wilcoxon Signed-Rank tests, are shown in Table 2. Comparisons between $d N, d S$, and $d N / d S$ of Ors and Grs using unpaired tests showed that Grs are more divergent than Ors in all three species (Table 3).

Genes with the highest and lowest $d N / d S$ values in $D$. suzukii provide insights into highly divergent or conserved functions. Among the most conserved Ors, Orco tops the list, followed by Or47a, Or92a, Or42b and Or24a, and whereas Gr21a, Gr28a, Gr28bB, Gr63a and Gr64c were the most conserved Grs. Most divergent Ors were Or19a, Or23a, Or69aA, Or65a and Or33a and the Grs included Gr10b, Gr93d, Gr92a, Gr85a and Gr22c (Additional file 2: Tables S1 and S2). This trend was comparable in D. takahashii and D. biarmipes.

\section{Selection}

Next we tested for the signatures of positive selection acting on a small proportion of sites that are often difficult to detect using the $d N / d S$ ratio across the entire gene. The adaptive branch-site random effects likelihood (aBSREL) approach [52] on homologous gene sets revealed two Ors and three Grs showing evidence of positive selection the suzukii-takahashii clade in the phylogenetic framework comprising nine melanogaster group drosophilids (Fig. 5; Additional file 2, Table S5). The number of tests for each gene set ranged from a small set of 11 (singletons with 
Table 3 Differences in substitution rates between Ors and Grs. Mean synonymous $(d S)$ and nonsynonymous ( $d M$ ) substitution rates and ratios (dN/dS) for Ors and Grs using D. melanogaster as an outgroup

\begin{tabular}{|c|c|c|c|c|c|c|c|c|c|}
\hline \multirow[b]{2}{*}{ Parameter } & \multicolumn{3}{|l|}{ Dsuz } & \multicolumn{3}{|l|}{ Dbia } & \multicolumn{3}{|l|}{ Dtak } \\
\hline & Ors & Grs & $p$-value & Ors & Grs & $p$-value & Ors & Grs & $p$-value \\
\hline$d N$ & 0.094 & 0.146 & 0.004 & 0.103 & 0.152 & 0.005 & 0.089 & 0.141 & 0.001 \\
\hline$d S$ & 0.936 & 1.036 & 0.924 & 1.009 & 1.039 & 0.681 & 0.945 & 1.018 & 0.623 \\
\hline$d N / d S$ & 0.102 & 0.141 & 0.005 & 0.104 & 0.142 & 0.002 & 0.093 & 0.131 & 0.001 \\
\hline
\end{tabular}

Mean $d N$ and $d N / d S$ was greater for Grs suggesting that, overall, Grs were more divergent than Ors in all three species

losses in some lineages) to as many as 43 for a gene with large expansion across spp. $(G r 59 d)$. A total of five lineages showed signatures of positive selection, four of those being at internal nodes and one being at an external node (Fig. 5). In all cases, the percentage of sites exhibiting signatures of positive selection $\left(\omega_{2} \%\right)$ was small, ranging from $1.1 \%$ to $7.2 \%$ (Fig. 5). Selection at the remaining sites $\left(\omega_{1}\right)$ ranged from very high purifying selection $\left(\omega_{1}<\right.$ $0.01)$ to neutral selection $\left(\omega_{1}=1\right)$.

In the suzukii-takahashii clade, positive selection was detected in Or2a, Gr5a and Gr97a along branches leading to both the suzukii and takahashii subgroups, while Gr58a showed signatures of positive selection along the branch leading to the suzukii subgroup (Fig. 5). In Or2a, positive selection was found at a very small percentage of codons $(1.9 \%)$, while the remaining sites exhibit signatures of purifying selection $\left(\omega_{1}=0.225\right)$ (Fig. 5). Strong purifying selection $\left(\omega_{1}<0.01\right)$ was evident at $96.4 \%$ of Gr5a, while $3.6 \%$ of the codons showed evidence of positive selection $\left(\omega_{2}=11 ; p=0.024\right)$. Gr58a also exhibited strong purifying selection $\left(\omega_{1}<0.01\right)$ at the majority of sites $(92.8 \%)$, while the remaining $7.2 \%$ exhibited signatures of positive selection $(; p=0.031)$. The vast proportion of Gr97a (98.6\%) shows no signs of selection pressure $\left(\omega_{1}=1.0\right)$ while $1.6 \%$ of the sites show evidence for positive selection $(p=0.004)$.

Next, the two largest expanded $\mathrm{Or}$ and $\mathrm{Gr}$ lineages were subjected to aBSREL analysis by restricting the phylogeny to the three species in the takahashii-suzukii clade. Only the Or67a lineage had genes with signatures of positive selection, of which two genes were in $D$. takahashii (DtakOr67a-4 and DtakOr67a-4) and one in D. suzukii (DsuzOr67a-3) (Fig. 6a). These results were independently confirmed using the branch-site test in PAML that further identified codons under positive selection (Fig. 6b).

Finally, we would like to state that the reason for reporting the less stringent p-values from HolmBonferroni corrections within gene sets was to extract candidates with some (any) evidence of positive selection.

\section{Discussion \\ Olfactory structures}

Peripheral olfactory structures in D. melanogaster have been studied over the years and have revealed stereotypic pattern of sensillary organization $[6,71,73]$. These studies laid a solid foundation to the functional mapping of sensilla $[74,75]$. More advanced molecular techniques have correlated the morphological and functional sensillary patterns with that of chemosensory gene expression $[76,77]$. A broadly conserved pattern emerged in our $D$. suzukii SEM studies as compared to D. melanogaster. Limited single sensillum recordings (SSR) from $D$. suzukii antennal basiconic (ab) sensilla in combination with high resolution gas chromatography (GC-SSR) suggested

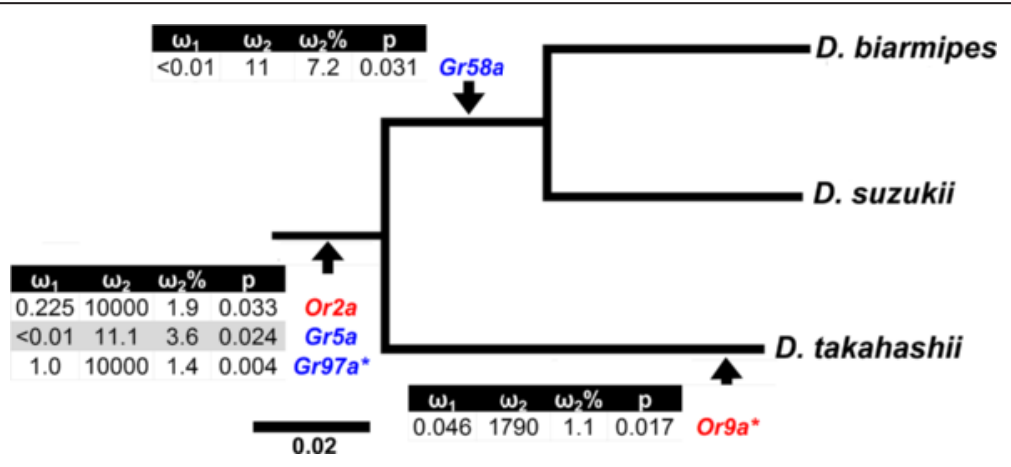

Fig. 5 Genes with signatures of positive selection in the suzukii-takahashii clade based on the adaptive branch-site random effects likelihood (aBSREL) method [52] performed across 59 Or and 61 Gr homologous gene sets within a phylogenetic framework comprised of nine melanogaster group Drosophila. P-values were corrected for multiple tests within each gene set using the Holm-Bonferroni method [63]. *Genes also showing signatures of positive selection using the branch site method in PAML [65] 

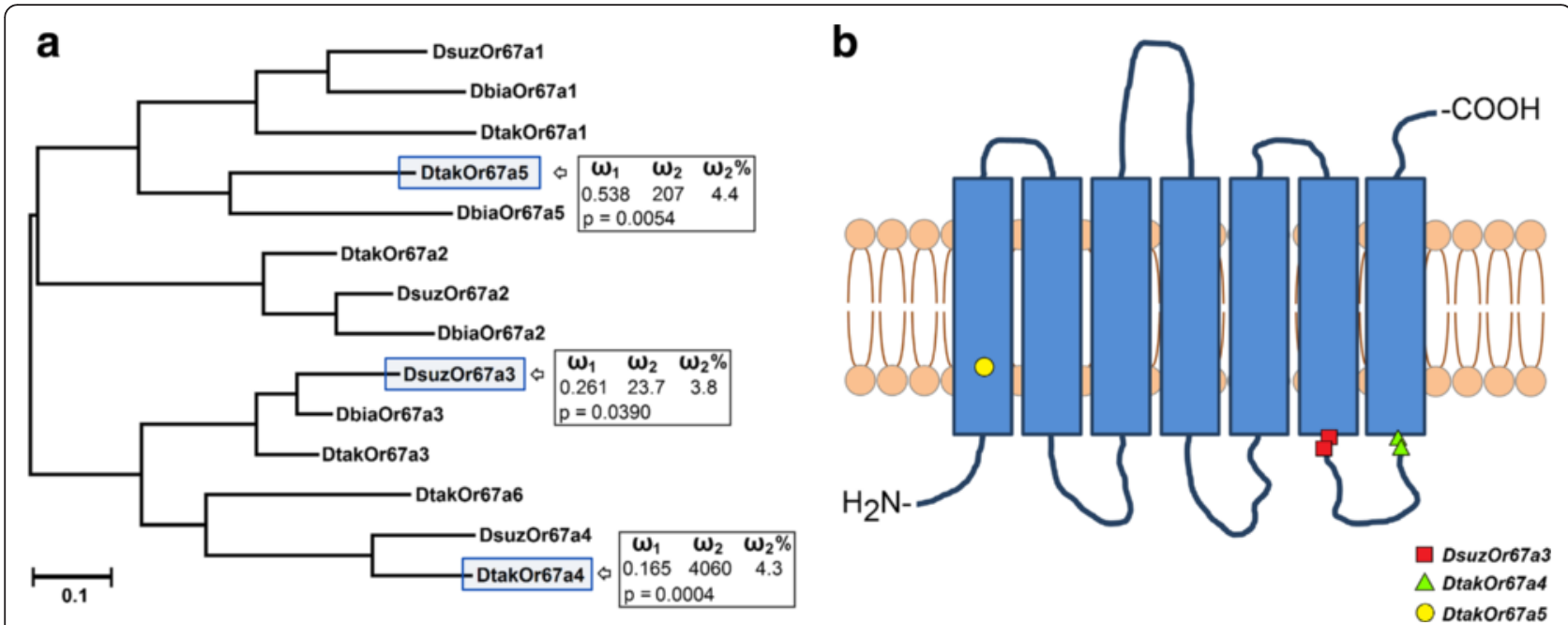

Fig. 6 Signatures of positive selection in the suzukii-takahashii Or67a lineage. a Three gene showed signatures of positive selection in the highly expanded Or67a lineage. Phylogeny adapted from Chiu et al. [35]. b The approximate position of the sites under positive selection based on branch site tests in PAML and the transmembrane domain predictions by OCTOPUS

a high conservation in response profile from the ab1 sensilla as compared to D. melanogaster, whereas other two large sensilla (ab2 and ab3) had significantly altered physiological profiles [39]. This could be due to the alteration in $\mathrm{Or}$ sequences and/or expression profiles.

\section{Repertoire size}

Unlike vertebrates and many insects, in which there has been extensive variation in the number of genes in the chemoreceptor families, the size of the Or and Gr repertoires in Drosophila have changed little during the last $\sim 70$ million years [78] despite their extensive distribution and diverse life history traits [79] that range from primitive sap and slime feeding (virilis-repleta) to more recent adaptations in the melanogaster group that utilize decaying and fermenting fruits [26]. Of 30 Drosophila genomes that have been sequenced, the melanogaster group is the most represented. Furthermore, this group has been well characterized in terms of chemosensory repertoire annotation.

A number of previous studies have described a balanced birth-and-death process of evolution, wherein the number of genes gained through duplication roughly equals the number of genes lost through pseudogenization, thus maintaining $\mathrm{Or}$ and $\mathrm{Gr}$ repertoires comprising approximately 60 genes each $[5,21,37,80]$. Our chemoreceptor annotations in the suzukii-takahashii clade revealed similar patterns (Fig. 4; Additional file 2: Tables S3 and S4). The size of the Or repertoires in D. suzukii and $D$. biarmipes are roughly the same as other melanogaster group flies, while D. takahashii has several additional Ors that make its repertoire the largest among all the annotated species in this group with 77 intact Ors. The size of the $G r$ repertoires in $D$. suzukii, D. biarmipes and D. takahashii are all relatively large compared to other melanogaster group members, with 81, 83 and 91 total proteins, respectively. Analysis of the evolutionary history of duplications and losses revealed that the expansions of the $\mathrm{Gr}$ lineages in the suzukii-takahashii clade occurred prior to $D$. suzukii's divergence from $D$. biarmipes, 7.3 mya [36]. Thus, the expanded $G r$ lineage is not a direct consequence of $D$. suzukii's adaptation to its expanded ecological niche, but could have simply helped facilitate the shift by providing ample variation for evolution to act upon.

\section{Expansions and losses}

Despite the maintenance of a standard repertoire size, gene births and deaths during trophic shifts can produce unique and rapidly evolving chemosensory repertoires. A study by McBride [19] showed that D. sechellia, a species endemic to the Seychelles and a specialist on the fruit of Morinda citrifolia, experienced an accelerated rate of chemoreceptor gene loss during its evolution to a specialist life style. A similar trend in the Grs was found in D. erecta, a specialist on Pandanus candelabrum [20]. A recent study further demonstrated a relationship between host-choice and chemoreceptor repertoire wherein four widely conserved Ors (Or9a, Or $22 a$, Or $42 b$ and $\mathrm{Or} 85 \mathrm{~d}$ ) that detect yeast-derived and fruit related compounds were uniquely lost in an herbivorous Drosophila, Scaptomyza flava, while Or67b, a receptor shown to enhance the sensitivity and detection of plant derived green leaf volatiles, was uniquely expanded [18]. These unique changes in the $\mathrm{Or}$ repertoire were considered as adaptive losses and gains towards the evolution of herbivory in Scaptomyza from its ancestral drosophilids that feed on yeast $[18,81]$. 
The D. suzukii and D. takahashii Or repertoires are distinct in having two large expansions, Or $23 a$ and Or67a, while only the Or67a expansion was retained in $D$. biarmipes. In D. melanogaster, Or $23 a$ is expressed on the surface of the B cell in antennal intermediate 2 (ai2) sensilla [82], formerly classified as antennal trichoid 2 (at2) sensilla [76]. And despite screening with a large panel of compounds using SSRs and the $\Delta$-halo system in D. melanogaster, no strong ligands for Or23a have been identified [83, 84]. In D. melanogaster, Or67a is expressed on the surface of the B cell in ab10 sensilla (Couto et al. 2005), where methyl benzoate and ethyl benzoate elicited strong excitatory

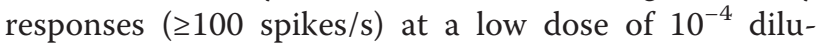
tions [83]. Five functional copies of Or67a in the $D$. suzukii strain from Italy have been found [40], while we identified only four intact copies and one pseudogene in the North American isolate, suggesting that the number of functional genes in the Or67a lineage can be variable across geographical regions. This group also suggested that $D$. suzukii's increased sensitivity to isoamyl acetate [33, 39], a yeast-derived and fresh fruit volatile, could be due to the expanded Or67a copy-numbers [40].

Interestingly, of the three species annotated here, $D$. suzukii's repertoire of Ors underwent the most gene deaths, with losses of Or74a, Or85a and Or98b. This results in the smallest number of $\mathrm{Or}$ lineages (51) among the nine drosophilids studied here (Additional file 2: Table S3). It is worth mentioning that this number of lineages is even smaller than D. sechellia's and $D$. erecta's, both of which have a very restricted diet. Of the three lost lineages, Or74a in D. melanogaster is a larval specific receptor expressed in a subset of ORNs in the larval dorsal organ (LDO) $[85,86]$ (Table 4). A heterologous expression using $\Delta$-halo system revealed excitatory responses to linear aliphatic compounds such as 1-hexanol, (E)-2-hexenal, 1-heptanol and 1-nonanol ( $\geq 100$ spikes/s), compounds commonly associated with fruits [86] (Table 4). The second, Or85a, is a narrowly tuned receptor expressed on the B cell of ab2 sensilla in D. melanogaster where ethyl 3hydroxybutyrate elicits a strong excitatory response [87]. Single sensillum recordings (SSRs) by Keesey et al. [39] showed similar response profiles for the B cell in ab2 sensilla in $D$. biarmipes and $D$. melanogaster, but not for $D$. suzukii. Ethyl 3-hydroxybutyrate still elicited a strong response, but 2-heptanone elicited the strongest response in D. suzukii (Table 4). However, 2-heptanone did not elicit a response in $D$. biarmipes or D. melanogaster, suggesting that a different, more broadly tuned Or is being expressed in D. suzukii's ab2 sensillum, which lends physiological evidence for the loss of Or85a from D. suzukii's repertoire of functional Ors. Very little is known

Table 4 Ligands and chemosensory organs, based on studies in D. melanogaster, are shown for lost and expanded lineages, and genes with signatures of positive selection ( $\mathrm{a}=$ antenna, $p=$ palp, $\mathrm{b}=$ basiconic, $\mathrm{t}=$ trichoid, $\mathrm{LDO}=$ larval dorsal organ)

\begin{tabular}{lllll}
\hline & Gene & Species & Ligands & Expression \\
\hline Losses & Or33cP & bia & Ethyl acetate, Cyclohexanone, Fenchone [100] & pb2A [76, 100] \\
& Or74aP & suz & E,E-2-4-nonadienal [101] & LDO [86] \\
& Or85aP & suz & Ethyl 3-hydroxybutyrate [87] & ab2B [76] \\
Or98bP & suz & unknown & ab6B*[76] \\
Expansions & Or23a & suz, tak & Isoamyl acetate [83] & ai2B [76] \\
& Or67a & suz, bia, tak & Ethyl benzoate, Methyl benzoate [83] & ab10B [76] \\
& Gr22a-f & tak & bitter compounds [102] & Labellum [103], larvae [104] legs [105] \\
& Gr36a-c & suz, bia, tak & bitter compounds [102] & Larvae [104], legs [105] \\
& Gr59ab & suz, bia, tak & bitter compounds [102] & Larvae [104], legs [105] \\
& Gr59cd & suz, bia, tak & bitter compounds [102] & Larvae $n$ [104], legs [105] \\
& Gr93a & suz & bitter compounds [102] & unknown \\
& Gr98b & suz & bitter compounds [102] & unknown \\
Gr98d & suz & bitter compounds [102] & Legs [105] \\
Or2a & suz, bia, tak & Ethyl 3-hydroxybutyrate, Isoamyl acetate [83] & ai3A [76] \\
Or9a & tak & 2-acetoin, 2,3-butanediol [84] & ab8B [76] \\
Gr5a & suz, bia, tak & Trehalose [93, 94] & Labellum [103], legs [105] \\
Gr58a & suz, bia & unknown & unknown \\
Gr97a & suz, bia, tak & unknown & Larvae [104]
\end{tabular}


about the function of $\mathrm{Or} 98 \mathrm{~b}$, except for its co-expression with $0 r 85 b$ in the A cell of the ab6 sensillum in D. melanogaster [76].

Finally, we made numerous attempts to sequence all three lost lineages in the $D$. suzukii genome. Our sequencing of Or74a and Or85a confirmed the highly degraded state of the loci in the North American isolate [35]. However, these two genes were considerably less degraded in the genome assembly from the Italian isolate, but pseudogenizations were still apparent [36]. Conversely, we were unsuccessful in sequencing the Or98b locus in D. suzukii. Amplicon size was consistent with that of a full length gene, but sequencing indicated that the locus is polymorphic in the North American assembly. However, we were able to build an intact gene model for $\mathrm{Or} 98 \mathrm{~b}$ in the genome assembly from the Italian assembly [36], and that sequence is provided in Additional file 1, Table S1. Polymorphism in Or98b among D. melanogaster strains was also reported, wherein several functional and pseudogene alleles were found in the Ives strain, a single pseudogene was found in the New Jersey strain, and no allele could not be amplified in the Oregon R strain [5].

\section{Divergence}

Measures of divergence provide insights into the molecular evolution which can often be correlated with conserved and divergent physiological processes. Our measure of divergence $(d N / d S)$ implies that both chemoreceptor families have evolved under strong purifying selection. However, these values are larger than the genome wide estimates of 0.095 for $\mathrm{X}$ chromosome genes and 0.090 for autosomal genes [35], demonstrating that these gene families are more divergent than average. Comparisons between $d N, d S$, and $d N / d S$ of Ors and Grs using unpaired tests showed that Grs are more divergent than Ors in all three species (Table 3).

Among the most conserved Ors, Orco tops the list, followed by Or47a, Or92a, Or42b and Or24a. These genes are also highly conserved in $D$. takahashii and $D$. biarmipes. Expression studies in D. melanogaster have revealed Orco to be a non-canonical receptor with a wide distribution $[9,10,77]$, whereas expression of the remaining Ors is confined to basiconic sensilla [76] except for Or24a which is larval specific in D. melanogaster [85]. Interestingly Or92a and Or42b are expressed in ab1 sensilla on the A and B ORNs, respectively. This high level of conservation corresponds with the electrophysiological data of ab1 that showed similar responses to a panel of ab1-sensitive odorants in D. melanogaster, D. biarmipes and D. suzukii [39]. An earlier study showed similar findings comparing nine species in the melanogaster subgroup [24]. Combined, these findings suggest that the role of ab1 sensilla has largely been conserved during at least the last $\sim 13$ million years of melanogaster group evolution. In fact, McBride and Arguello [20] proposed this phenomenon to be applicable for all the large basiconic sensilla (ab1-3) in five members of the melanogaster subgroup.

On the other hand, the expression of the most divergent receptors in D. suzukii is predicted to be among three different sensilla types. Of these, both Or19a and Or23a are expressed in intermediate sensilla [71, 82], Or33a and Or69aA are restricted to a basiconic [71, 82], and Or65a is expressed in a trichoid [76]. Potential response characteristic and the significance of these Ors in D. suzukii remains an exciting avenue to explore. Three of these five homologues in $D$. melanogaster (Or23a, Or65a, and Or69aA) did not respond with high sensitivity to any of the odorants tested heterologously [83]. Physiological data is lacking for DmelOr33a. Two different studies reported DmelOr19a responding to limonene, a major citrus fruit volatile [83, 88].

Among the gustatory receptors in the suzukii-takahashii clade, Gr21a was the most conserved, surpassing even Orco. The other highly conserved Grs include $G r 28 b B, G r 28 a$, Gr63a and Gr64c. It is worth mentioning that Gr21a and Gr63a are highly conserved among insects $[5,80]$, and together confer the sensitivity to carbon dioxide [89, 90], whereas $G r 28 b B$ and Gr28a are part of the bitter receptor family and are shown to be ubiquitously expressed in a wide array of sensory and non-sensory tissue [91, 92]. The five most divergent Grs include Gr10b, Gr93d, Gr92a, Gr85a and $G r 22 c$; little is known about their expression or response characteristics.

\section{Selection}

Our set of 11 chemoreceptor lineages with signatures of positive selection in the nine species is smaller than the reported 20 in an earlier study that compared chemosensory repertoires in 12 Drosophila, even though two genes (Or9a and Gr5a) were common in both studies [17]. These differences could be due to multiple reasons. Our study focused on the drosophilids from the melanogaster group that have a relatively comparable host range [26], while the other study included six species outside the melanogaster group. In addition, we adjusted the $p$-values based on more stringent Holm-Bonferroni corrections which reduced the number of significant candidates. However, we note that our corrections were performed within, but not across gene sets; therefore, these results should be interpreted with caution.

Of the 11 genes, we found four genes (Or2a, Gr5a, Gr58a and Gr97a) that were significant in the branches leading to $D$. suzukii. In $D$. melanogaster, Or $2 a$ is expressed in ai3 sensilla [76, 82] and has been shown to respond to ethyl 3-hydroxybutyrate and isoamyl acetate 
eliciting only moderate responses ( 50 spikes/s) [83]. It is interesting to note that isoamyl acetate has been identified as a strong ligand from suzukii-associated yeasts [33] and host fruits [40]. Among the Grs, DmelGr5 has been studied in detail. Molecular, physiological and behavioral studies identified it as a sugar receptor with a strong selectivity and sensitivity to trehalose [93, 94]. Importance of sugars in D. suzukii is more pronounced since this fly also uses a variety of non-conventional sugar sources such as nectar and cherry blossom in the field [95]. Functional data on DmelGr58a and DmelGr97a is lacking [91]. Our restricted aBSREL analysis of the four largest expanded lineages (Or23a, Or67a, Gr59a and Gr59d) in the suzukii-takahashii clade revealed evidence for positive selection only in Or67a, where three copies showed signatures of positive selection (Fig. 6a). Overall, adaptation of D. suzukii to novel niches appears to be facilitated by unique expansions and losses of chemosensory lineages. Together with our earlier that described the volatile chemical landscapes of D. suzukii [33], present study further provides novel insights into the synchronous evolution of signaling and reception in flies.

\section{Conclusions}

We manually annotated the olfactory and gustatory receptor families of the pest fly, D. suzukii to complement our earlier analysis of the evolution of olfactory signals in this fly that showed salience of a set of yeast derived odorants enriched in the D. suzukii landscape [33]. We further annotated two close relatives, $D$. biarmipes and D. takahashii to compare and contrast their chemosensory repertoire with that of $D$. suzukii. This revealed three unique losses of Ors (Or74a, Or85, Or98b) in D. suzukii among the three species in the suzukii-takahashii clade, and two large expansions in the olfactory receptors, Or23a and Or67a. There was an overall pattern of purifying selection in both chemoreceptor families, with Ors exhibiting greater conservation. The gustatory genome repertoire size in this clade was by far the largest among all the annotated species of the melanogaster group. Finally, our analysis for the signature of positive episodic selection in $D$. suzukii led to the identification of Or2a and one copy of Or67a as strong candidates. Taken together, this study provides detailed insights into the molecular evolution of the two major chemoreceptor families in an invasive and pestiferous fly. The evolution of a serrated ovipositor for piercing the skin of fresh fruits is a unique innovation that conferred a distinct advantage in fruit flies to exploit fruits of varying ripeness. In tephritids, this innovation facilitated the radiation of thousands of species [96]. Surprisingly, this innovation exists in only two known drosophilids, D. suzukii and D. subpulchrella, both of which are members of the suzukii subgroup [31]. The recent sequencing of $D$. suzukii (pest) and D. biarmipes (non-pest) within the suzukii subgroup provided us with an excellent opportunity to explore the contribution of chemosensation in the evolution of pestilence in D. suzukii.

\section{Additional files}

Additional file 1: Table S1. D. suzukii Or coding and peptide sequences. Table S2. D. suzukii Gr coding and peptide sequences. Table S3. D. biarmipes Or coding and peptide sequences. Table S4. D. biarmipes Gr coding and peptide sequences. Table S5. D. takahashii Or coding and peptide sequences. Table S6. D. takahashii Gr coding and peptide sequences. (XLSX $379 \mathrm{~kb}$ )

Additional file 2: Table S1. Non-synonymous $(d N)$ and synonymous (dS) substation rates and ratios (dN/dS) of Ors in members of the suzukiitakahashii clade using D. melanogaster as an outgroup. Table S2. Non-synonymous $(d N)$ and synonymous $(d S)$ substation rates and ratios $(d N / d S)$ of Grs in members of the suzukii-takahashii clade using D. melanogaster as an outgroup. Table S3. The number of functional Or genes in each orthologous group for nine species in the melanogaster group based on Almeida et al. [50]. Table S4. The number of functional Gr genes in each orthologous group for nine species in the melanogaster group based on Almeida et al. [50]. Table S5. Summary of the chemoreceptors in the suzukii-takahashii clade under episodic positive selection as revealed by aBSREL analyses. (XLSX $35 \mathrm{~kb}$ )

Additional file 3: Or alignment file (Phylip). Olfactory receptor peptide sequences of Drosophila suzukii (Dsuz), D. biarmipes (Dbia), D. takahashii (Dtak) and D. melanogaster (Dmel) $\geq 360$ aa in length were multiply aligned using MUSCLE v3.8.31. (PHY 229 kb)

Additional file 4: Or phylogenetic tree file (Nexus). Phylogenetic analysis of Ors in Drosophila suzukii (Dsuz), D. biarmipes (Dbia), D. takahashii (Dtak) and D. melanogaster (Dmel) using a Maximum Likelihood method. Evolutionary history was inferred using a Maximum Likelihood method based on the JTT matrix-based model. The tree was constructed using RAxML under the JTT model of substitution with NNI topology search, based on an amino acid alignment by MUSCLE. Branch support was estimated using 500 bootstrap replications. The tree is rooted with Orco. (NEX $26 \mathrm{~kb}$ )

Additional file 5: Gr alignment file (Phylip). Gustatory receptor peptide sequences of Drosophila suzukii (Dsuz), D. biarmipes (Dbia), D. takahashii (Dtak) and D. melanogaster (Dmel) $\geq 340$ aa in length were multiply aligned using MUSCLE v3.8.31. (PHY 274 kb)

Additional file 6: Gr phylogenetic tree file (Nexus). Phylogenetic analysis of Grs in Drosophila suzukii (Dsuz), D. biarmipes (Dbia), D. takahashii (Dtak) and D. melanogaster (Dmel) using a Maximum Likelihood method. Evolutionary history was inferred using a Maximum Likelihood method based on the JTT matrix-based model. The tree was constructed using RAxML under the JTT model of substitution with NNI topology search, based on an amino acid alignment by MUSCLE. Branch support was estimated using 500 bootstrap replications. The tree is rooted with Gr21a. (NEX $31 \mathrm{~kb}$ )

Additional file 7: Figure S1. Evolutionary history of duplications and losses in four expanded lineages in the chemoreceptor families based on the parsimony-based method of gene tree reconciliation in NOTUNG V2.8.1.6 [51]. (TIF $2581 \mathrm{~kb})$

\section{Abbreviations}

aBSREL, adaptive branch-site random effects likelihood; Gr, gustatory receptor; LRT, likelihood ratio tests; Or, odorant receptor; Orco, olfactory receptor co-receptor; ORN, olfactory receptor neuron; SEM, scanning electron microscopy; VOC, volatile organic compounds 


\section{Acknowledgements}

The authors thank Frank Zalom and Johanna Chiu (both at the University of California, Davis), Michael Prender, Yiyuan Li and RJ Nowling (all at the University of Notre Dame) for advice and helpful discussions. We gratefully acknowledge Hugh Robertson (The University of Illinois at UrbanaChampaign) for much discussions, advice, and exchange of ideas. We also thank Julien Pelletier (Keele University, UK), Johanna Chiu (UC-Davis), and Carolyn (Lindy) McBride (Princeton University) for constructive comments on an earlier version of this manuscript.

\section{Funding}

This work was supported by grants to ZS from the California Cherry Marketing and Research Board grant (G00001951), UC Davis-Notre Dame Research Agreement award (201224810), and USDA National Institute for Food and Agriculture (NIFA) Specialty Crops Research Initiative (SCRI) Award (2015-51181-24252) as a subcontract between the University of Notre Dame and North Carolina State University.

\section{Availability of data and material}

All relevant data are available within the manuscript and its additional files (Additional file 1 has Table S1 through S6 that contain all the annotated Or and $\mathrm{Gr}$ coding and peptide sequences for D. suzukii, D. biarmipes and D. takahashii. Additional file 3, 4, 5 and 6 are aligned peptide sequence and phylogeny tree files for Or and Grs, respectively. The phylogenetic data and the support files have been deposited at the: http://datadryad.org/ review?doi=doi:10.5061/dryad.5q1 h4view?doi=doi:10.5061/dryad.5q1h4.

\section{Authors' contributions}

$\mathrm{PVH}$ carried out the gene annotation and molecular evolution analysis and drafted the manuscript. CLR participated in the phylogenetic and molecular evolution analysis. CMJ and GJR assisted in PCR amplifications and sequencing. MS participated in early annotations of $D$. suzukii receptors. ZS had the research idea, and participated in developing the study design and drafting the manuscript along with PVH. All authors read and approved the final manuscript.

\section{Competing interests}

The authors declare that they have no competing interests.

\section{Consent for publication}

Not applicable.

\section{Ethics approval and consent to participate}

Not applicable.

\section{Received: 28 May 2016 Accepted: 29 July 2016}

Published online: 17 August 2016

\section{References}

1. Benton R, Vannice KS, Gomez-Diaz C, Vosshall LB. Variant ionotropic glutamate receptors as chemosensory receptors in Drosophila. Cell. 2009; 136(1):149-62.

2. Rytz R, Croset V, Benton R. lonotropic receptors (IRs): chemosensory ionotropic glutamate receptors in Drosophila and beyond. Insect Biochem Mol Biol. 2013;43(9):888-97.

3. Hansson BS, Stensmyr MC. Evolution of insect olfaction. Neuron. 2011;72(5): 698-711.

4. Joseph RM, Carlson JR. Drosophila Chemoreceptors: A Molecular Interface Between the Chemical World and the Brain. Trends Genet. 2015;31(12): 683-95.

5. Robertson HM, Warr CG, Carlson JR. Molecular evolution of the insect chemoreceptor gene superfamily in Drosophila melanogaster. Proc Natl Acad Sci U S A. 2003;100:14537-42.

6. Stocker RF. The organization of the chemosensory system in Drosophila melanogaster - a review. Cell Tissue Res. 1994;275(1):3-26.

7. Ache BW, Young JM. Olfaction: diverse species, conserved principles. Neuron. 2005;48(3):417-30.

8. Hildebrand JG, Shepherd GM. Mechanisms of olfactory discrimination: converging evidence for common principles across phyla. Ann Rev Neurosci. 1997;20:595-631.
9. Benton R, Sachse S, Michnick SW, Vosshall LB. Atypical membrane topology and heteromeric function of Drosophila odorant receptors in vivo. Plos Biol. 2006;4(2):240-57.

10. Larsson MC, Domingos Al, Jones WD, Chiappe ME, Amrein H, Vosshall LB. Or83b encodes a broadly expressed odorant receptor essential for Drosophila olfaction. Neuron. 2004;43(5):703-14.

11. Zhang $\mathrm{H}-\mathrm{J}$, Anderson AR, Trowell SC, Luo AR, Xiang Z-H, Xia Q-Y. Topological and Functional Characterization of an Insect Gustatory Receptor. PLoS One. 2011;6(8), e24111.

12. Macharia R, Mireji P, Murungi E, Murilla G, Christoffels A, Aksoy S, Masiga D. Genome-Wide Comparative Analysis of Chemosensory Gene Families in Five Tsetse Fly Species. PLoS Negl Trop Dis. 2016;10(2), e0004421.

13. Obiero GF, Mireji PO, Nyanjom SR, Christoffels A, Robertson HM, Masiga DK. Odorant and gustatory receptors in the tsetse fly Glossina morsitans morsitans. PLoS Negl Trop Dis. 2014;8(4), e2663.

14. Engsontia P, Sanderson AP, Cobb M, Walden KKO, Robertson HM, Brown S. The red flour beetle's large nose: An expanded odorant receptor gene family in Tribolium castaneum. Insect Biochem Mol Biol. 2008;38(4):387-97.

15. Robertson HM, Wanner KW. The chemoreceptor superfamily in the honey bee, Apis mellifera: Expansion of the odorant, but not gustatory, receptor family. Genome Res. 2006;16(11):1395-403.

16. Zhou X, Rokas A, Berger SL, Liebig J, Ray A, Zwiebel LJ. Chemoreceptor Evolution in Hymenoptera and Its Implications for the Evolution of Eusociality. Genome Biol Evol. 2015;7(8):2407-16.

17. Gardiner A, Barker D, Butlin RK, Jordan WC, Ritchie MG. Drosophila chemoreceptor gene evolution: selection, specialization and genome size. Mol Ecol. 2008;17(7):1648-57.

18. Goldman-Huertas B, Mitchell RF, Lapoint RT, Faucher CP, Hildebrand JG, Whiteman NK. Evolution of herbivory in Drosophilidae linked to loss of behaviors, antennal responses, odorant receptors, and ancestral diet. Proc Natl Acad Sci U S A. 2015;112(10):3026-31.

19. McBride CS. Rapid evolution of smell and taste receptor genes during host specialization in Drosophila sechellia. Proc Natl Acad Sci U S A. 2007;104(12): 4996-5001.

20. McBride CS, Arguello JR. Five drosophila genomes reveal nonneutral evolution and the signature of host specialization in the chemoreceptor superfamily. Genetics. 2007;177(3):1395-416.

21. Nozawa M, Nei M. Evolutionary dynamics of olfactory receptor genes in Drosophila species. Proc Natl Acad Sci U S A. 2007;104(17):7122-7.

22. Dekker T, Ibba I, Siju KP, Stensmyr MC, Hansson BS. Olfactory shifts parallel superspecialism for toxic fruit in Drosophila melanogaster sibling, D. sechellia. Curr Biol. 2006;16(1):101-9.

23. Linz J, Baschwitz A, Strutz A, Dweck HKM, Sachse S, Hansson BS, Stensmyr MC. Host plant-driven sensory specialization in Drosophila erecta. P Roy Soc B-Biol Sci. 2013;280(1760):20130626.

24. Stensmyr MC, Dekker T, Hansson BS. Evolution of the olfactory code in the Drosophila melanogaster subgroup. P Roy Soc B-Biol Sci. 2003;270(1531): 2333-40.

25. Syed Z. Chemical ecology and olfaction in arthropod vectors of diseases. Curr Opin Insect Sci. 2015;10:83-9.

26. Markow TA. The secret lives of Drosophila flies. eLife. 2015;4.

27. Vosshall LB. Into the mind of a fly. Nature. 2007;450(7167):193-7.

28. Rota-Stabelli O, Blaxter M, Anfora G. Drosophila suzukii. Curr Biol. 2013;23(1): R8-9.

29. Walsh DB, Bolda MP, Goodhue RE, Dreves AJ, Lee JC, Bruck DJ, Walton VM, O'Neal SD, Zalom FG. Drosophila suzukii (Diptera: Drosophilidae): invasive pest of ripening soft fruit expanding its geographic range and damage potential. J Integr Pest Manag. 2011;2(1):G1-7.

30. Markow TA, O'Grady PM. Drosophila: a guide to species identification and use. London: Academic; 2006.

31. Atallah J, Teixeira L, Salazar R, Zaragoza G, Kopp A. The making of a pest: the evolution of a fruit-penetrating ovipositor in Drosophila suzukii and related species. P Roy Soc B-Biol Sci. 2014;281 (1781):20132840.

32. Cini $A$, loriatti $C$, Anfora $G$. A review of the invasion of Drosophila suzukii in Europe and a draft research agenda for integrated pest management. Bull Insectology. 2012;65(1):149-60.

33. Scheidler NH, Liu C, Hamby KA, Zalom FG, Syed Z. Volatile codes: Correlation of olfactory signals and reception in Drosophila-yeast chemical communication. Sci Rep. 2015;5:14059. 
34. Hamby KA, Becher PG. Current knowledge of interactions between Drosophila suzukii and microbes, and their potential utility for pest management. J Pest Sci. 2016;1-10.

35. Chiu JC, Jiang $X$, Zhao L, Hamm CA, Cridland JM, Saelao P, Hamby KA Lee EK, Kwok RS, Zhang G, et al. Genome of Drosophila suzukii, the Spotted Wing Drosophila. G3. 2013;3(12):2257-71.

36. Ometto L, Cestaro A, Ramasamy S, Grassi A, Revadi S, Siozios S, Moretto M, Fontana P, Varotto C, Pisani D, et al. Linking Genomics and Ecology to Investigate the Complex Evolution of an Invasive Drosophila Pest. Genome Biol Evol. 2013;5(4):745-57.

37. Guo S, Kim J. Molecular evolution of Drosophila odorant receptor genes. Mol Biol Evol. 2007;24(5):1198-207.

38. Dekker T, Revadi S, Mansourian S, Ramasamy S, Lebreton S, Becher PG, Angeli S, Rota-Stabelli O, Anfora G. Loss of Drosophila pheromone reverses its role in sexual communication in Drosophila suzukii. Proc R Soc Lond B Biol Sci. 2015;282(1804):20143018

39. Keesey IW, Knaden M, Hansson BS. Olfactory Specialization in Drosophila suzukii Supports an Ecological Shift in Host Preference from Rotten to Fresh Fruit. J Chem Ecol. 2015;41(2):121-8

40. Revadi S, Vitagliano S, Stacconi MVR, Ramasamy S, Mansourian S, Carlin S, Vrhovsek U, Becher PG, Mazzoni V, Rota-Stabelli O, et al. Olfactory responses of Drosophila suzukii females to host plant volatiles. Physiol Entomol. 2015; 40(1):54-64.

41. dos Santos G, Schroeder AJ, Goodman JL, Strelets VB, Crosby MA, Thurmond J, Emmert DB, Gelbart WM, FlyBase C. FlyBase: introduction of the Drosophila melanogaster Release 6 reference genome assembly and large-scale migration of genome annotations. Nucleic Acids Res. 2015; 43(Database issue):D690-697.

42. Corpet F. Multiple sequence alignment with hierarchical-clustering. Nucleic Acids Res. 1988;16(22):10881-90.

43. Reese MG, Eeckman FH, Kulp D, Haussler D. Improved splice site detection in Genie. J Comput Biol. 1997:4(3):311-23.

44. Stothard P. The sequence manipulation suite: JavaScript programs for analyzing and formatting protein and DNA sequences. BioTechniques. 2000;28(6):1102. 1104

45. Gasteiger E, Gattiker A, Hoogland C, Ivanyi I, Appel RD, Bairoch A. ExPASy: The proteomics server for in-depth protein knowledge and analysis. Nucleic Acids Res. 2003;31(13):3784-8.

46. Doyle JJ. A rapid DNA isolation procedure for small quantities of fresh leaf tissue. Phytochem Bull. 1987:19:11-5.

47. Untergasser A, Cutcutache I, Koressaar T, Ye J, Faircloth BC, Remm M, Rozen SG. Primer3-new capabilities and interfaces. Nucleic Acids Res. 2012;40(15), e115.

48. Drosophila Odorant Receptor Nomenclature Committee. A unified nomenclature system for the Drosophila odorant receptors. Cell. 2000; 102(2):145-46.

49. Viklund H, Elofsson A. OCTOPUS: improving topology prediction by twotrack ANN-based preference scores and an extended topological grammar. Bioinformatics (Oxford, England). 2008;24(15):1662-8.

50. Almeida FC, Sanchez-Gracia A, Luis Campos J, Rozas J. Family Size Evolution in Drosophila Chemosensory Gene Families: A Comparative Analysis with a Critical Appraisal of Methods. Genome Biol Evol. 2014;6(7):1669-82.

51. Stolzer M, Lai H, Xu M, Sathaye D, Vernot B, Durand D. Inferring duplications, losses, transfers and incomplete lineage sorting with nonbinary species trees. Bioinformatics (Oxford, England). 2012;28(18):1409-15.

52. Smith MD, Wertheim JO, Weaver S, Murrell B, Scheffler K, Kosakovsky Pond SL. Less is more: an adaptive branch-site random effects model for efficient detection of episodic diversifying selection. Mol Biol Evol. 2015;32(5):1342-53.

53. Venables WN, Ripley BD. Modern Applied Statistics with S. 4th ed. New York: Springer; 2002.

54. Sievers F, Wilm A, Dineen D, Gibson TJ, Karplus K, Li W, Lopez R, McWilliam $\mathrm{H}$, Remmert M, Soeding J, et al. Fast, scalable generation of high-quality protein multiple sequence alignments using Clustal Omega. Mol Syst Biol. 2011;7.

55. McWilliam H, Li W, Uludag M, Squizzato S, Park YM, Buso N, Cowley AP, Lopez R. Analysis Tool Web Services from the EMBL-EBI. Nucleic Acids Res. 2013;41(W1):W597-600.

56. Nei M, Gojobori T. Simple methods for estimating the numbers of synonymous and nonsynonymous nucleotide substitutions. Mol Biol Evol. 1986;3(5):418-26.
57. Korber B. HIV Signature and Sequence Variation Analysis. In: Rodrigo AG, Learn GH, editors. Computational Analysis of HIV Molecular Sequences. Dordrecht: Kluwer Academic Publishers; 2000. p. 55-72.

58. Katoh K, Standley DM. MAFFT multiple sequence alignment software version 7: improvements in performance and usability. Mol Biol Evol. 2013; 30(4):772-80.

59. Jones DT, Taylor WR, Thornton JM. The rapid generation of mutation data matrices from protein sequences. Comput Appl Biosci. 1992;8(3): 275-82.

60. Tamura K, Stecher G, Peterson D, Filipski A, Kumar S. MEGA6: Molecular Evolutionary Genetics Analysis version 6.0. Mol Biol Evol. 2013;30(12):2725-9.

61. Suyama M, Torrents D, Bork P. PAL2NAL: robust conversion of protein sequence alignments into the corresponding codon alignments. Nucleic Acids Res. 2006;34(Web Server issue):W609-612.

62. Kosakovsky Pond SL, Posada D, Gravenor MB, Woelk CH, Frost SD. GARD: a genetic algorithm for recombination detection. Bioinformatics (Oxford, England). 2006;22(24):3096-8.

63. Holm S. A simple sequentially rejective multiple test procedure. Scand J Stat. 1979:6(2):65-70.

64. Benjamini $Y$, Hochberg $Y$. Controlling the false discovery rate - a practical and powerful approach to multiple testing. J R Stat Soc Ser B Methodol. 1995;57(1):289-300.

65. Yang Z. PAML: a program package for phylogenetic analysis by maximum likelihood. Comput Appl Biosci. 1997;13(5):555-6.

66. Yang Z, Wong WS, Nielsen R. Bayes empirical Bayes inference of amino acid sites under positive selection. Mol Biol Evol. 2005;22(4):1107-18.

67. Edgar RC. MUSCLE: multiple sequence alignment with high accuracy and high throughput. Nucleic Acids Res. 2004;32(5):1792-7.

68. Stamatakis A. RAxML version 8: a tool for phylogenetic analysis and postanalysis of large phylogenies. Bioinformatics. 2014;30(9):1312-13.

69. Miller MA, Pfeiffer W, Schwartz T. The CIPRES science gateway: enabling high-impact science for phylogenetics researchers with limited resources. Proceedings of the 1st Conference of the Extreme Science and Engineering Discovery Environment: Bridging from the eXtreme to the campus and beyond. Chicago: ACM; 2012. p. 1-8.

70. Rambaut A. FigTree, a graphical viewer of phylogenetic trees. 2007. http:// tree.bio.ed.ac.uk/software/figtree.

71. Shanbhag SR, Muller B, Steinbrecht RA. Atlas of olfactory organs of Drosophila melanogaster - 1. Types, external organization, innervation and distribution of olfactory sensilla. Int J Insect Morphol Embryol. 1999;28(4): 377-97.

72. Steinbrecht RA. Structure and function of insect olfactory sensilla. CIBA Found Symp. 1996:200:158-74. discussion 174-157.

73. Carlson JR. Olfaction in Drosophila: From odor to behavior. Trends Genet. 1996;12(5):175-80.

74. de Bruyne M, Clyne PJ, Carlson JR. Odor coding in a model olfactory organ: the Drosophila maxillary palp. J Neurosci. 1999:19(11):4520-32.

75. de Bruyne M, Foster K, Carlson JR. Odor coding in the Drosophila antenna. Neuron. 2001:30(2):537-52.

76. Couto A, Alenius M, Dickson BJ. Molecular, anatomical, and functional organization of the Drosophila olfactory system. Curr Biol. 2005;15(17):153547.

77. Vosshall LB, Amrein H, Morozov PS, Rzhetsky A, Axel R. A spatial map of olfactory receptor expression in the Drosophila antenna. Cell. 1999;96(5): 725-36.

78. Nei M, Niimura Y, Nozawa M. The evolution of animal chemosensory receptor gene repertoires: roles of chance and necessity. Nat Rev Genet. 2008:9(12):951-63.

79. Markow TA, O'Grady PM. Evolutionary genetics of reproductive behavior in Drosophila: connecting the dots. Annu Rev Genet. 2005;39:263-91.

80. Robertson HM, Kent LB. Evolution of the gene lineage encoding the carbon dioxide receptor in insects. J Insect Sci (Online). 2009:9:19.

81. Whiteman NK, Pierce NE. Delicious poison: genetics of Drosophila host plant preference. Trends Ecol Evol. 2008:23(9):473-8.

82. Lin CC, Potter CJ. Re-Classification of Drosophila melanogaster Trichoid and Intermediate Sensilla Using Fluorescence-Guided Single Sensillum Recording. PLoS One. 2015;10(10), e0139675.

83. Hallem EA, Carlson JR. Coding of odors by a receptor repertoire. Cell. 2006; 125(1):143-60

84. Hallem EA, Ho MG, Carlson JR. The molecular basis of odor coding in the drosophila antenna. Cell. 2004;117(7):965-79. 
85. Fishilevich E, Domingos Al, Asahina K, Naef F, Vosshall LB, Louis M. Chemotaxis behavior mediated by single larval olfactory neurons in Drosophila. Curr Biol. 2005;15(23):2086-96.

86. Kreher SA, Kwon JY, Carlson JR. The molecular basis of odor coding in the Drosophila larva. Neuron. 2005;46(3):445-56.

87. Stensmyr MC, Giordano E, Balloi A, Angioy AM, Hansson BS. Novel natural ligands for Drosophila olfactory receptor neurones. J Exp Biol. 2003; 206(Pt 4):715-24.

88. Dweck HK, Ebrahim SA, Farhan A, Hansson BS, Stensmyr MC. Olfactory proxy detection of dietary antioxidants in Drosophila. Curr Biol. 2015;25(4): 455-66.

89. Jones WD, Cayirlioglu P, Kadow IG, Vosshall LB. Two chemosensory receptors together mediate carbon dioxide detection in Drosophila. Nature. 2007:445(7123):86-90.

90. Kwon JY, Dahanukar A, Weiss LA, Carlson JR. The molecular basis of CO2 reception in Drosophila. Proc Natl Acad Sci U S A. 2007;104(9):3574-8.

91. Freeman EG, Dahanukar A. Molecular neurobiology of Drosophila taste. Curr Opin Neurobiol. 2015;34:140-8.

92. Thorne N, Amrein H. Atypical expression of Drosophila gustatory receptor genes in sensory and central neurons. J Comp Neurol. 2008;506(4):548-68.

93. Chyb S, Dahanukar A, Wickens A, Carlson JR. Drosophila Gr5a encodes a taste receptor tuned to trehalose. Proc Natl Acad Sci U S A. 2003;100 Suppl 2:14526-30.

94. Dahanukar A, Foster K, van Naters W, Carlson JR. A Gr receptor is required for response to the sugar trehalose in taste neurons of Drosophila. Nat Neurosci. 2001;4(12):1182-6.

95. Tochen S, Walton V, Lee J. Impact of floral feeding on adult Drosophila suzukii survival and nutrient status. J Pest Sci. 2016.

96. Díaz-Fleischer F, Papaj DR, Prokopy RJ, Norrbom AL, Aluja M. Evolution of Fruit Fly Oviposition Behavior. In: Fruit Flies (Tephritidae): Phylogeny and Evolution of Behavior. Aluja M, Norrbom AL, editors. CRC Press; 2001: 811-849.

97. Clyne PJ, Warr CG, Carlson JR. Candidate taste receptors in Drosophila. Science. 2000;287(5459):1830-4.

98. Clyne PJ, Warr CG, Freeman MR, Lessing D, Kim JH, Carlson JR. A nove family of divergent seven-transmembrane proteins: Candidate odorant receptors in Drosophila. Neuron. 1999;22(2):327-38.

99. Dunipace L, Meister S, McNealy C, Amrein H. Spatially restricted expression of candidate taste receptors in the Drosophila gustatory system. Curr Biol. 2001;11(11):822-35.

100. Goldman AL, Van der Goes van Naters W, Lessing D, Warr CG, Carlson JR. Coexpression of two functional odor receptors in one neuron. Neuron. 2005;45(5):661-6.

101. Mathew D, Martelli C, Kelley-Swift E, Brusalis C, Gershow M, Samuel AD, Emonet T, Carlson JR. Functional diversity among sensory receptors in a Drosophila olfactory circuit. Proc Natl Acad Sci U S A. 2013;110(23): E2134-2143.

102. Weiss LA, Dahanukar A, Kwon JY, Banerjee D, Carlson JR. The Molecular and Cellular Basis of Bitter Taste in Drosophila. Neuron. 2011;69(2):258-72.

103. Thorne N, Chromey C, Bray S, Amrein H. Taste perception and coding in Drosophila. Curr Biol. 2004;14(12):1065-79.

104. Kwon JY, Dahanukar A, Weiss LA, Carlson JR. Molecular and Cellular Organization of the Taste System in the Drosophila Larva. J Neurosci. 2011;31(43):15300-9.

105. Ling F, Dahanukar A, Weiss LA, Kwon JY, Carlson JR. The Molecular and Cellular Basis of Taste Coding in the Legs of Drosophila. J Neurosci. 2014; 34(21):7148-64.

\section{Submit your next manuscript to BioMed Central and we will help you at every step:}

- We accept pre-submission inquiries

- Our selector tool helps you to find the most relevant journal

- We provide round the clock customer support

- Convenient online submission

- Thorough peer review

- Inclusion in PubMed and all major indexing services

- Maximum visibility for your research

Submit your manuscript at www.biomedcentral.com/submit

C Biomed Central 